\title{
"Intelligent Ensemble" Projections of Precipitation and Surface Radiation in support of Agricultural Climate Change Adaptation
}

Patrick C. Taylor and Noel C. Baker NASA Langley Research Center

Climate Science Branch

4 May 2015

Special Acknowledgement: Noel Baker, NASA Postdoctoral Researcher This presentation is heavily drawn from her research. 


\section{Motivation: Climate influences Society}
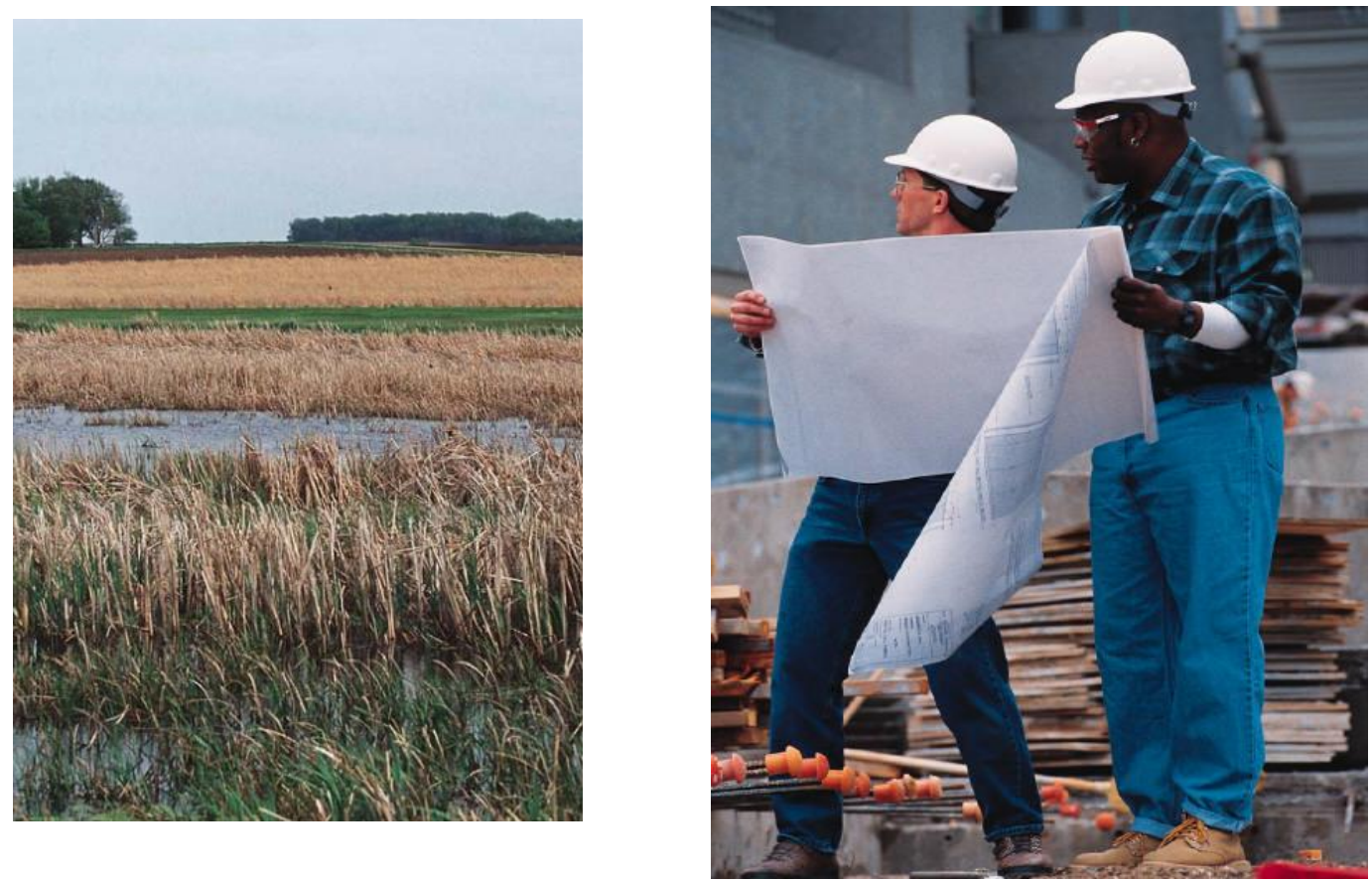

A location climate influences

-Agriculture

-Energy needs

-Water availability

- Infrastructure

-Building codes
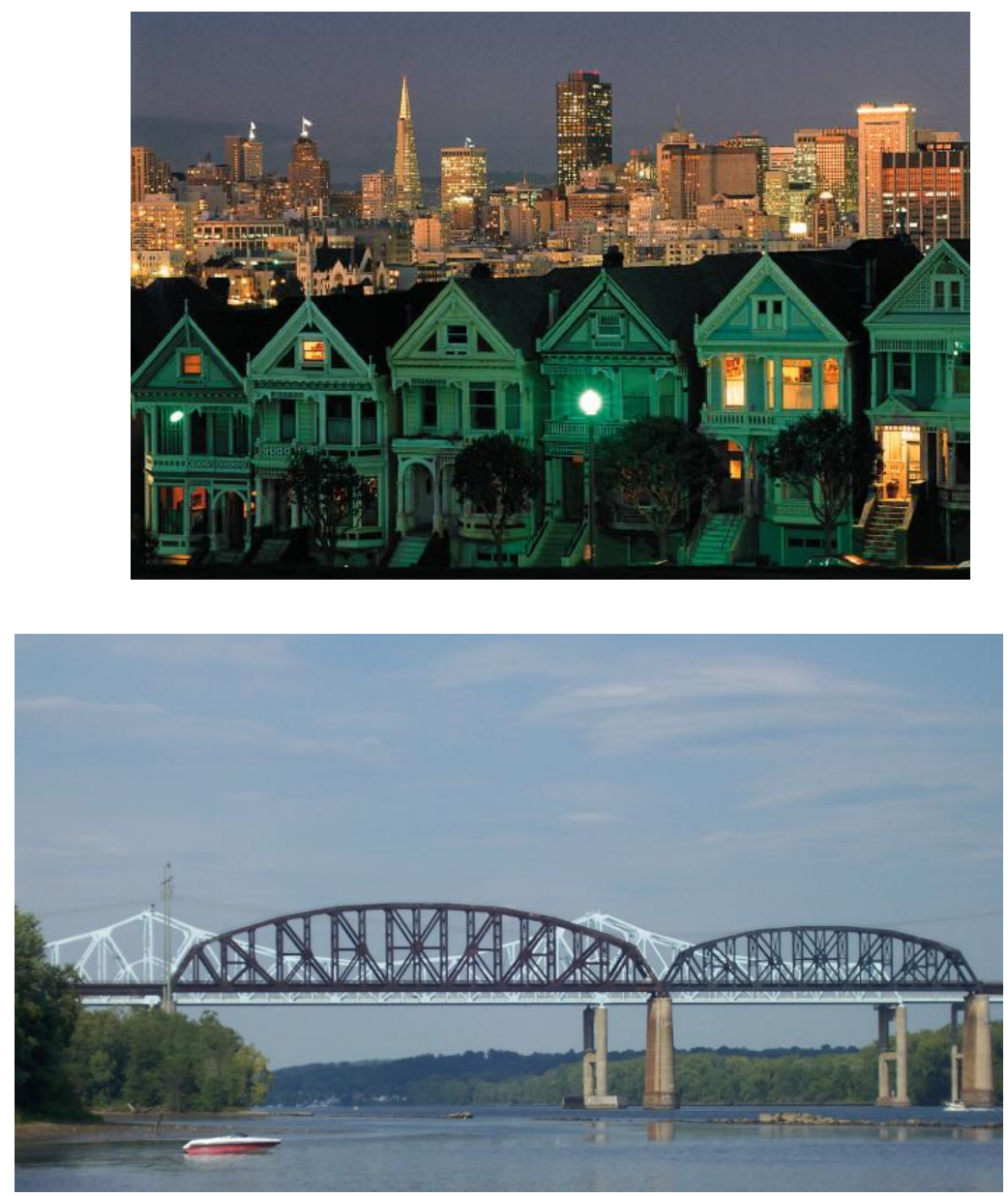


\section{Earth's climate is changing.}

Data source: NASA's Goddard Institute for Space Studies

(GISS). Credit: NASA/GISS

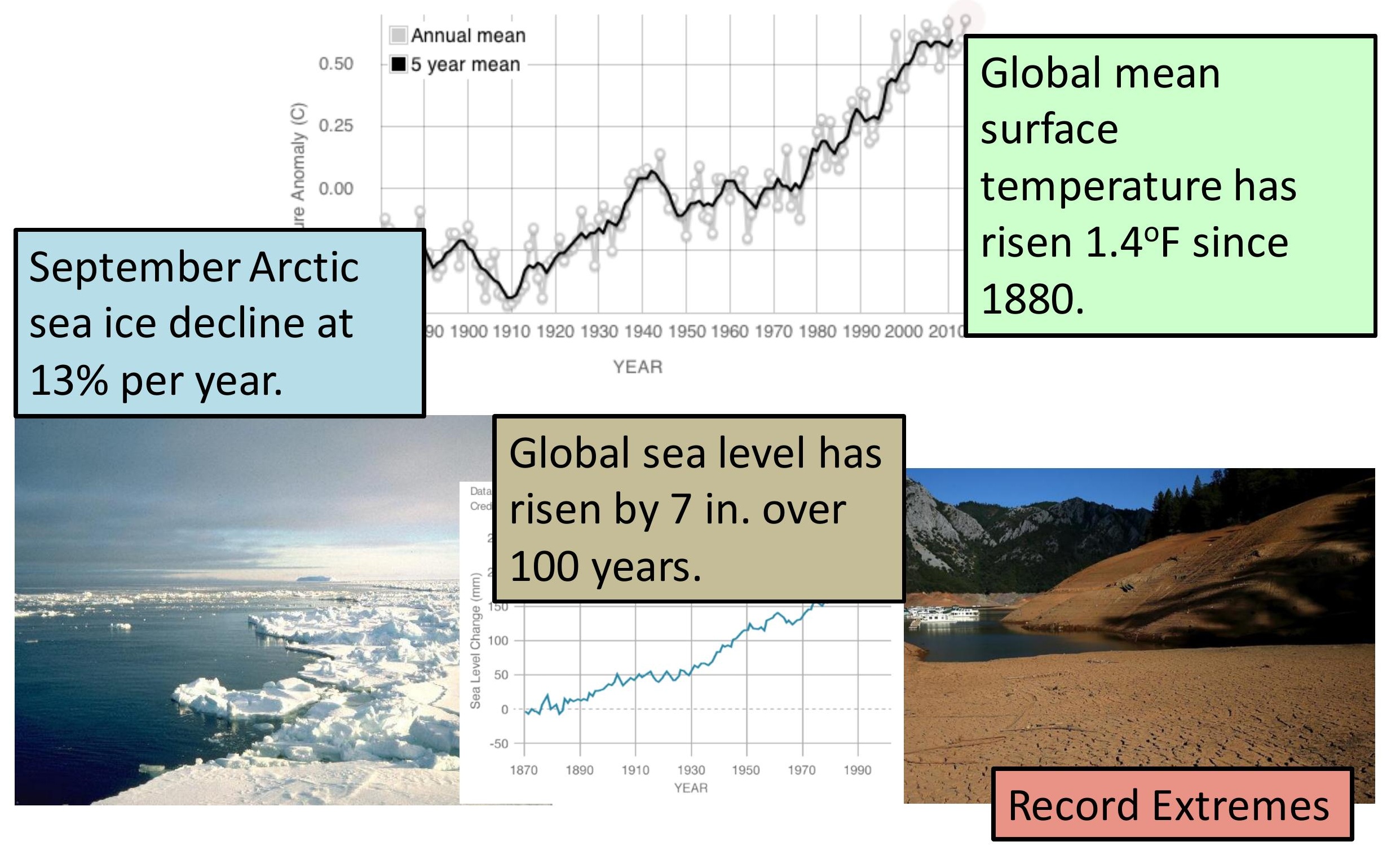




\section{Adaptation Planning is required}

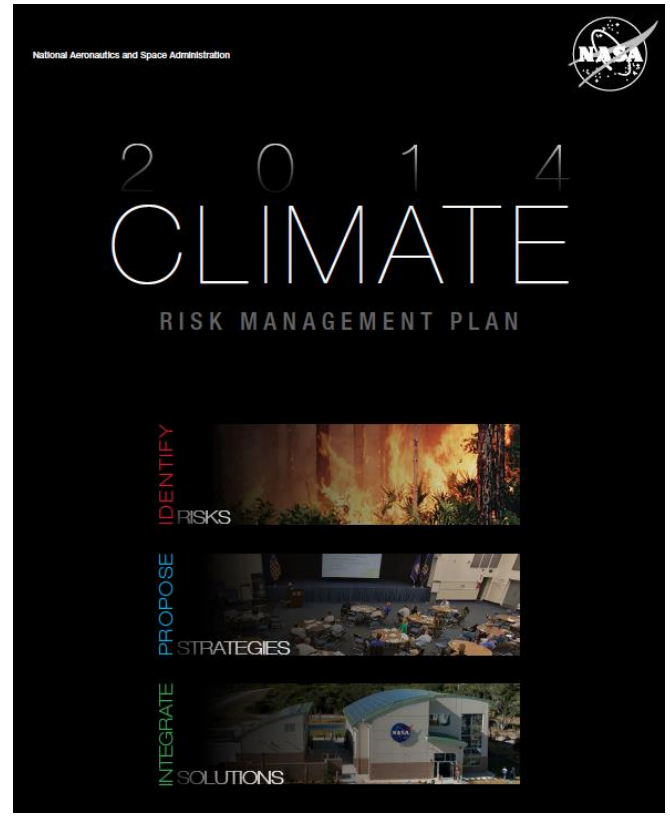
GOVERNOR'S COMMISSION ON CLIMATE CHANGE

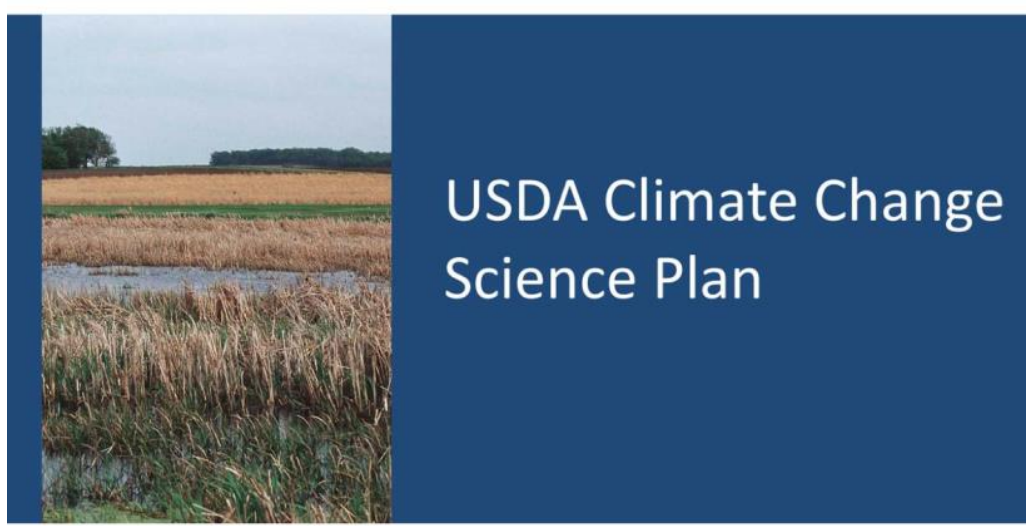

Final Report: A Climate Change Action Plan

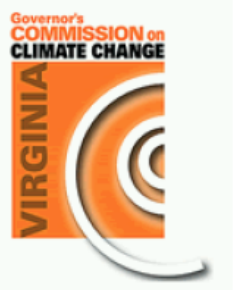

December 15, 2008

The Honorable L. Preston Bryant, Jr. Secretary of Natural Resources

\section{Climate projections are necessary.}




\section{Coupled Model}

Intercomparison

Project 5

(CMIP5)

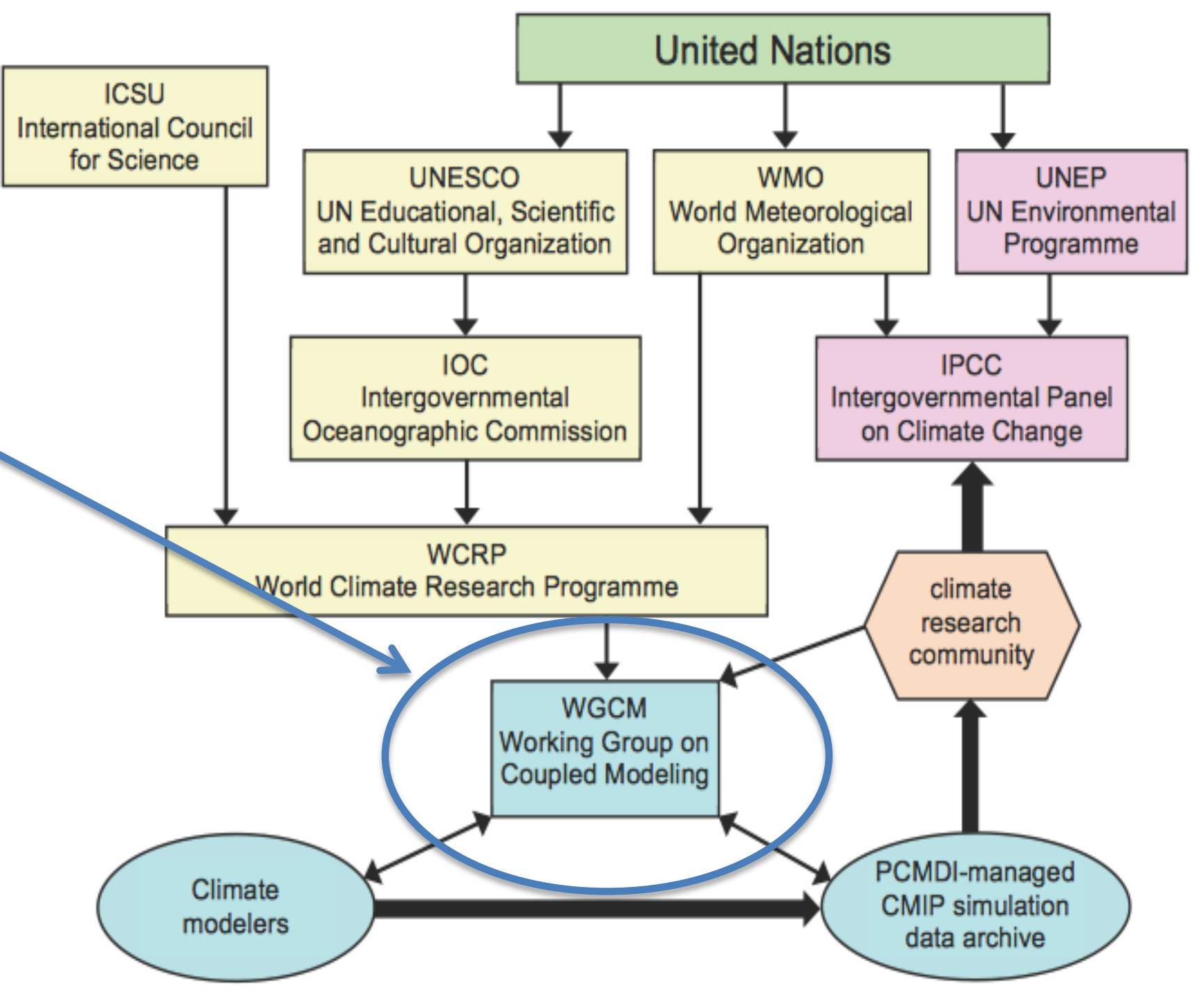

Taylor et al. (2012; BAMS)

FIG. I. The relationship of CMIP5 to organizations established to coordinate climate research activities internationally and to the IPCC, the modeling centers, and the climate research community. 


\section{Expected Changes: Constructing climate projections}

(a) Global average surface temperature change

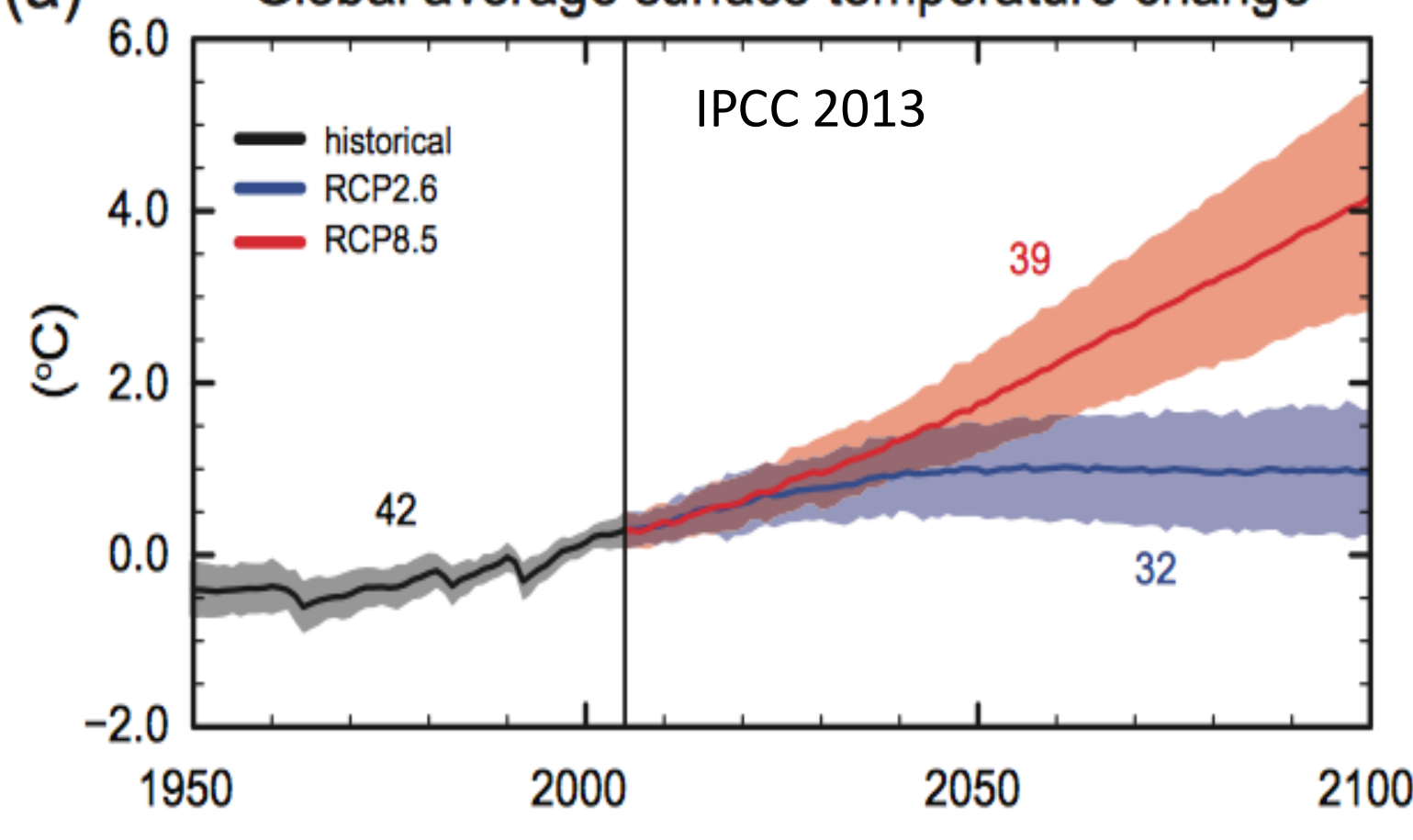

Mean over

2081-2100

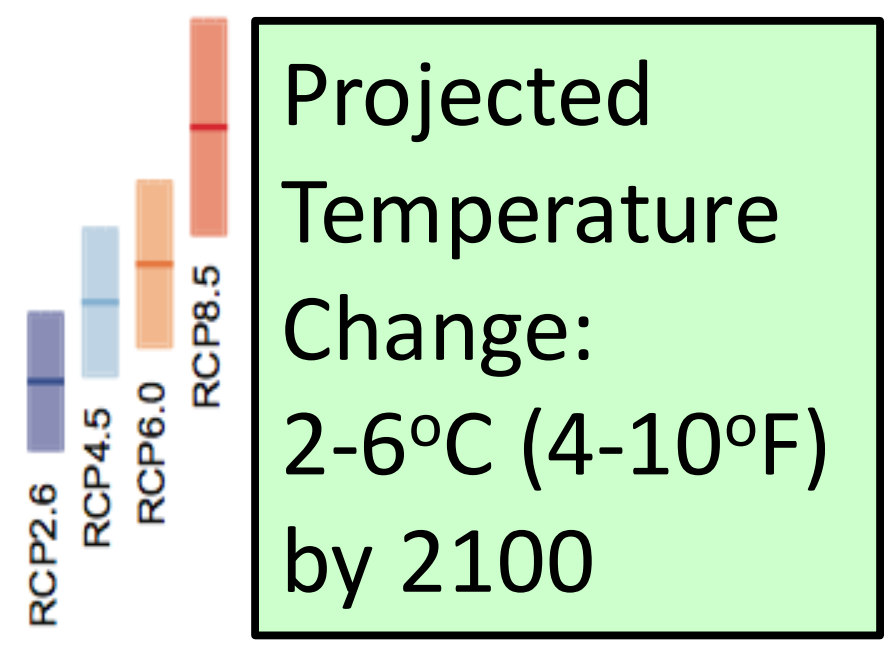

Conventional Ensemble Projection Approach: One model, one vote 


\section{Conventional vs. "Intelligent" Ensemble Method}

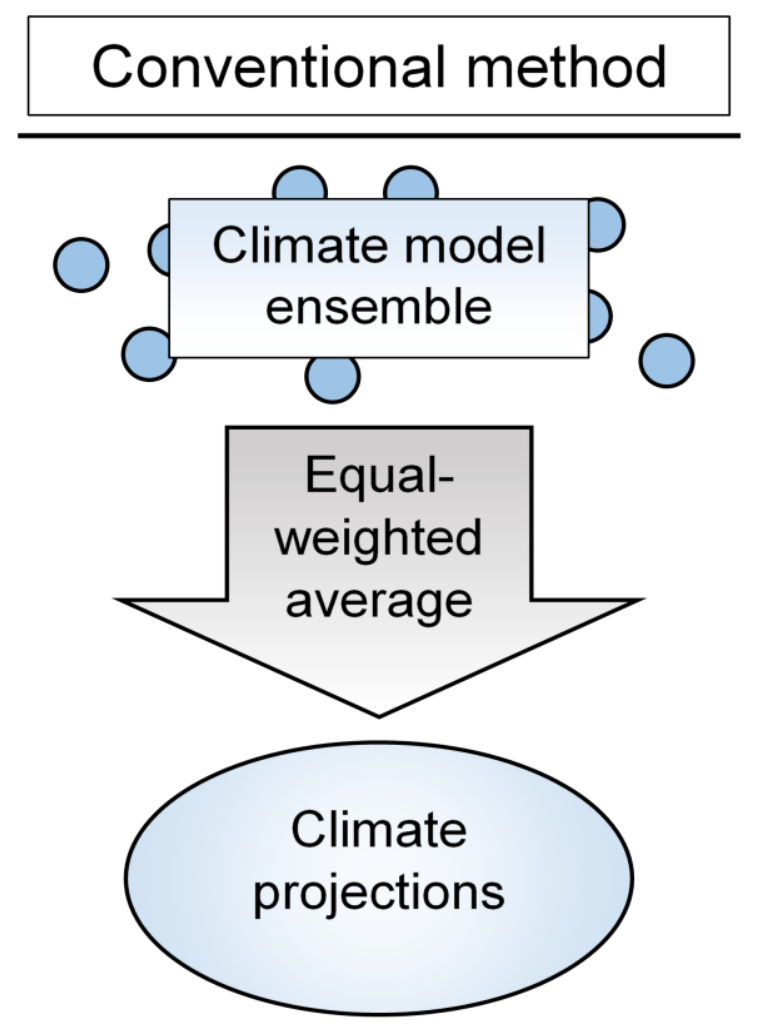

New methodology synergistically uses NASA observations and model strengths and weaknesses to improve climate projections.
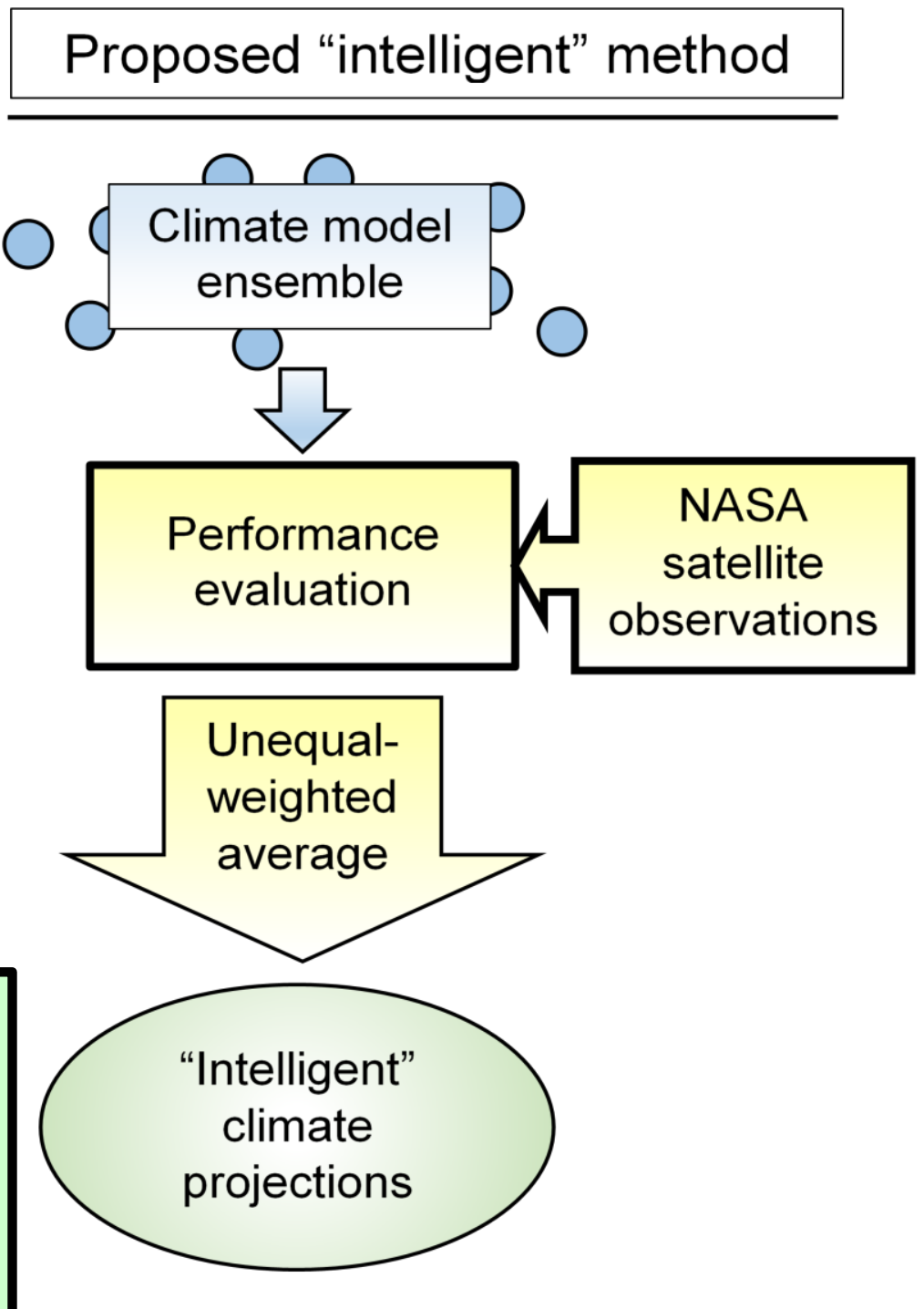


\section{NASA Earth Science Missions Current \& Planned}

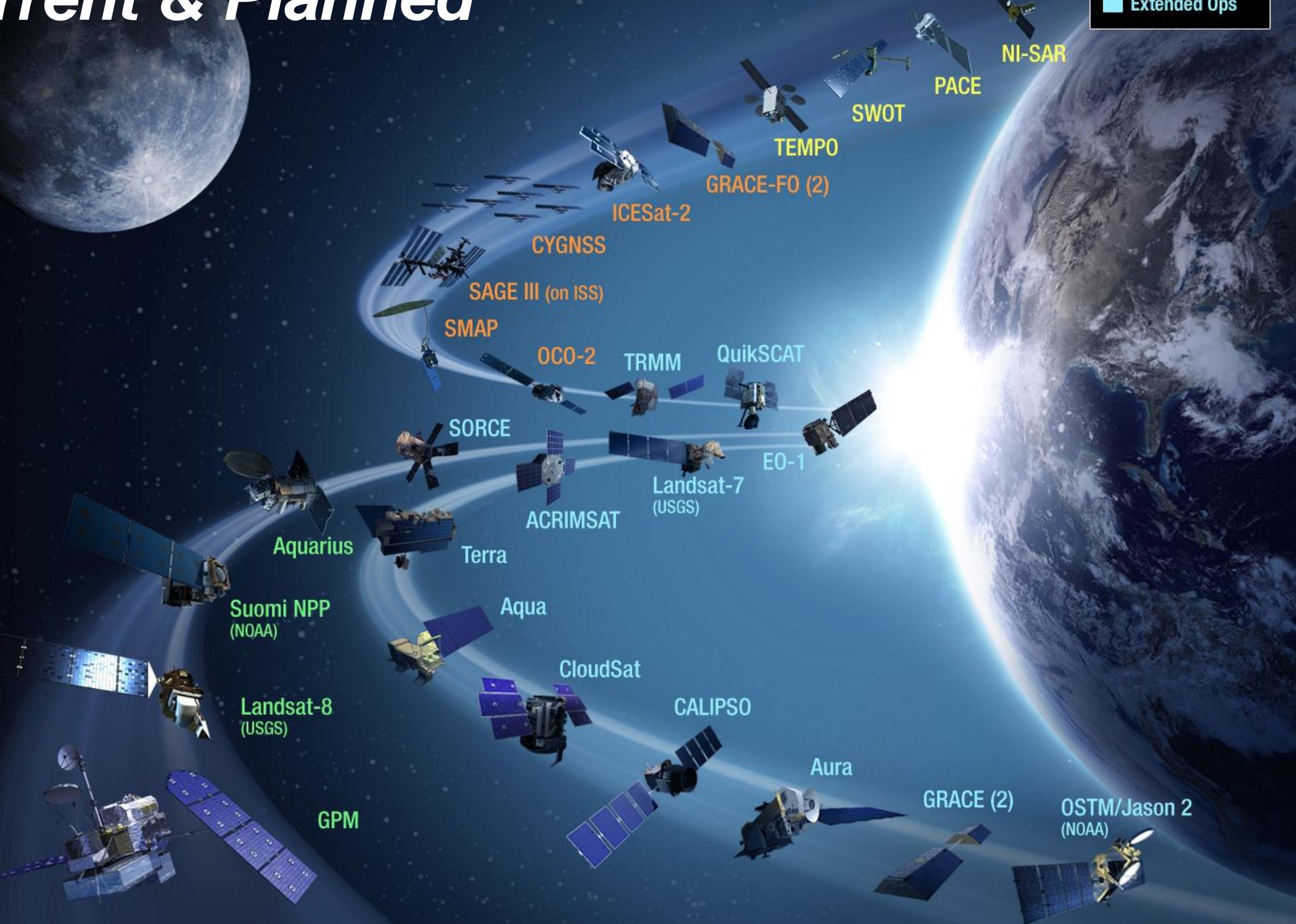




\section{Metric Selection: Earth's Climate is determine by energy flows}

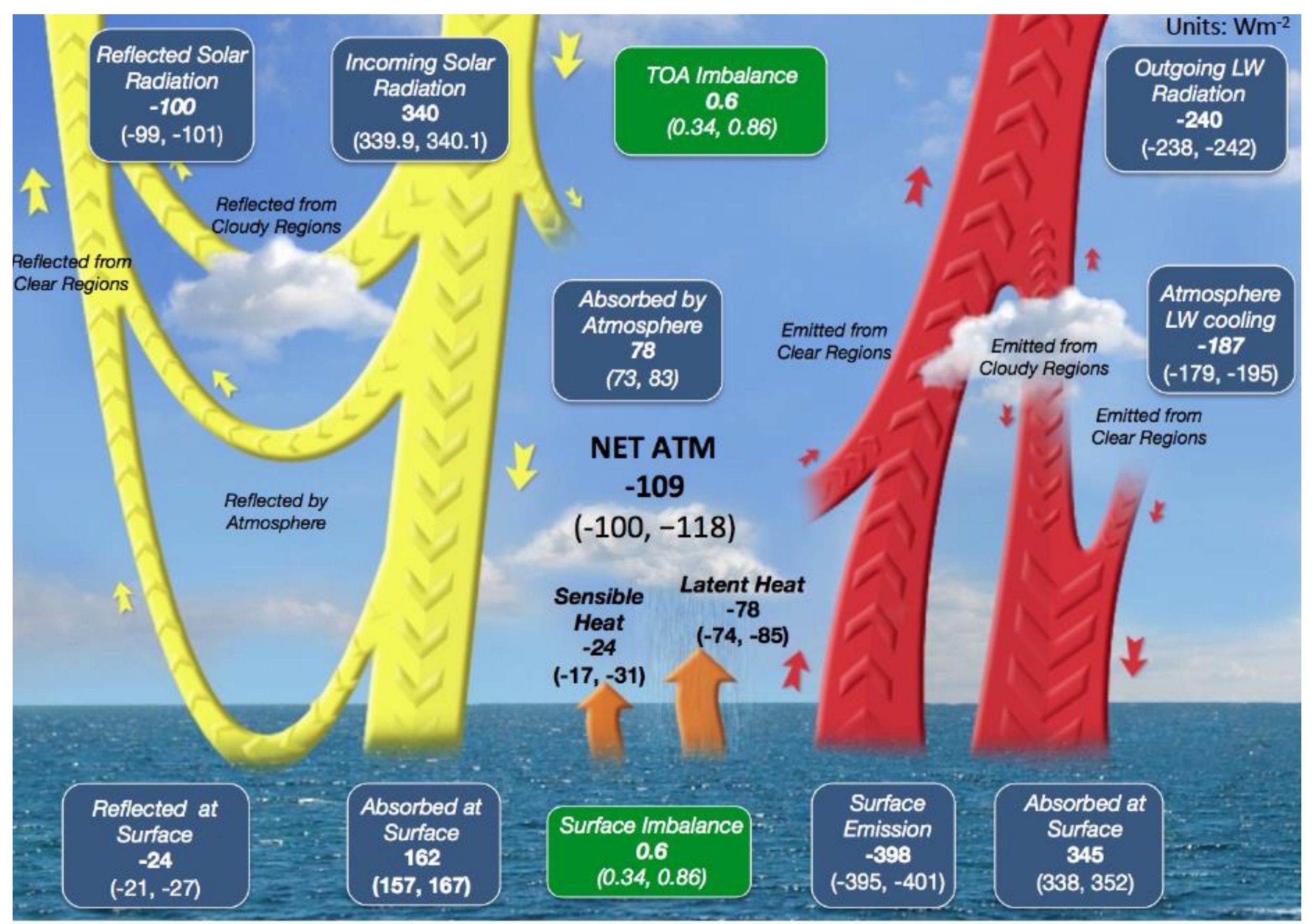




\section{Methodology}

- Use perfect model approach to determine the quantities whose performance in an unforced variability simulation robustly relates to climate projections

- Then use NASA observations to produce dataconstrained climate projections

- The climate model ensemble is used to understand the relationship between variability in Earth's energy budget and the sensitivity of Earth's climate to a radiative perturbation. 
OLR all-sky variance test OLR all-sky K-S test OLR all-sky local variance test OLR all-sky EMD value OLR cloudy-sky variance test OLR cloudy-sky K-S test OLR cloudy-sky local variance test

OLR cloudy-sky EMD value

OLR clear-sky variance test OLR clear-sky K-S test OLR clear-sky local variance test

OLR clear-sky EMD value SW all-sky variance test SW all-sky K-S test SW all-sky local variance test SW all-sky EMD value

SW cloudy-sky variance test SW cloudy-sky K-S test SW cloudy-sky local variance test SW cloudy-sky EMD value SW clear-sky variance test SW clear-sky K-S test SW clear-sky local variance test SW clear-sky EMD value Surface temperature variance test Surface temperature K-S test Surface temperature local variance test

Surface temperature EMD value OLR/Ts variance test

OLR(cloudy-sky)/Ts variance test OLR/Ts K-S test

OLR(cloudy-sky)/Ts K-S test

OLR Ts regression means test OLR(cloudy-sky) Ts regression means test SW/Ts variance test

$\mathrm{SW}$ (cloudy-sky)/Ts variance test SW/Ts K-S test

SW(cloudy-sky)/Ts K-S test SW Ts regression means test SW(cloudy-sky) Ts regression means test
Metric mean

Performance Evaluation

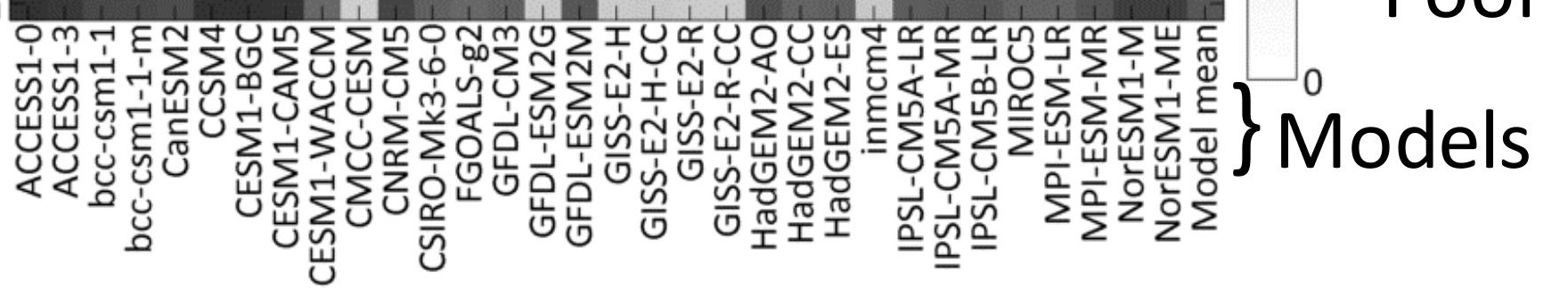


Producing "Intelligent Ensemble Projections: Selecting "Ideal" Metrics:

Best metrics have both a low standard deviation and $I^{2}$ value.

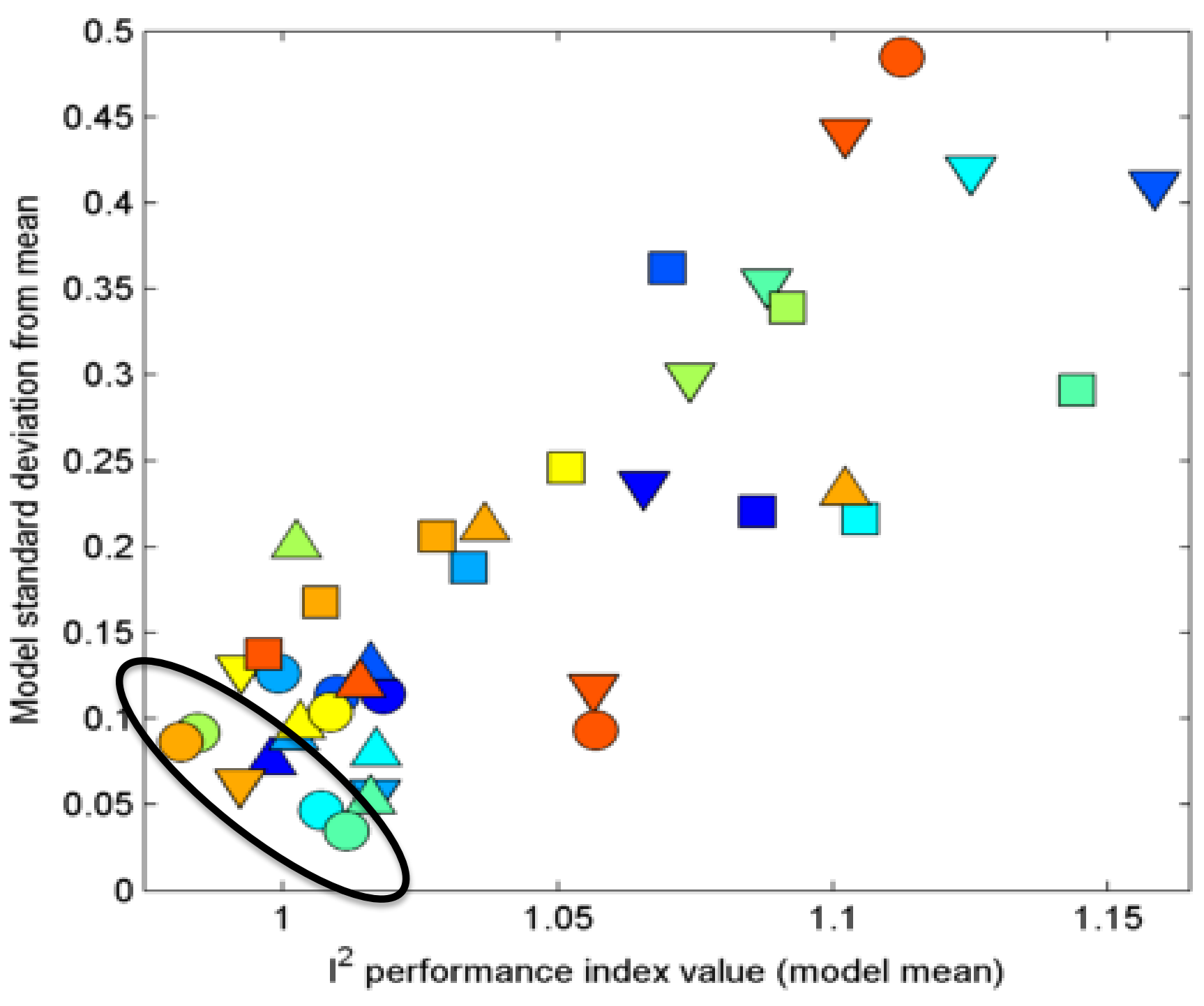




\section{Results: $21^{\text {st }}$-century "Intelligent" projections (regional weights)}

"Intelligent" ensemble mean precipitation trend (cm/year)

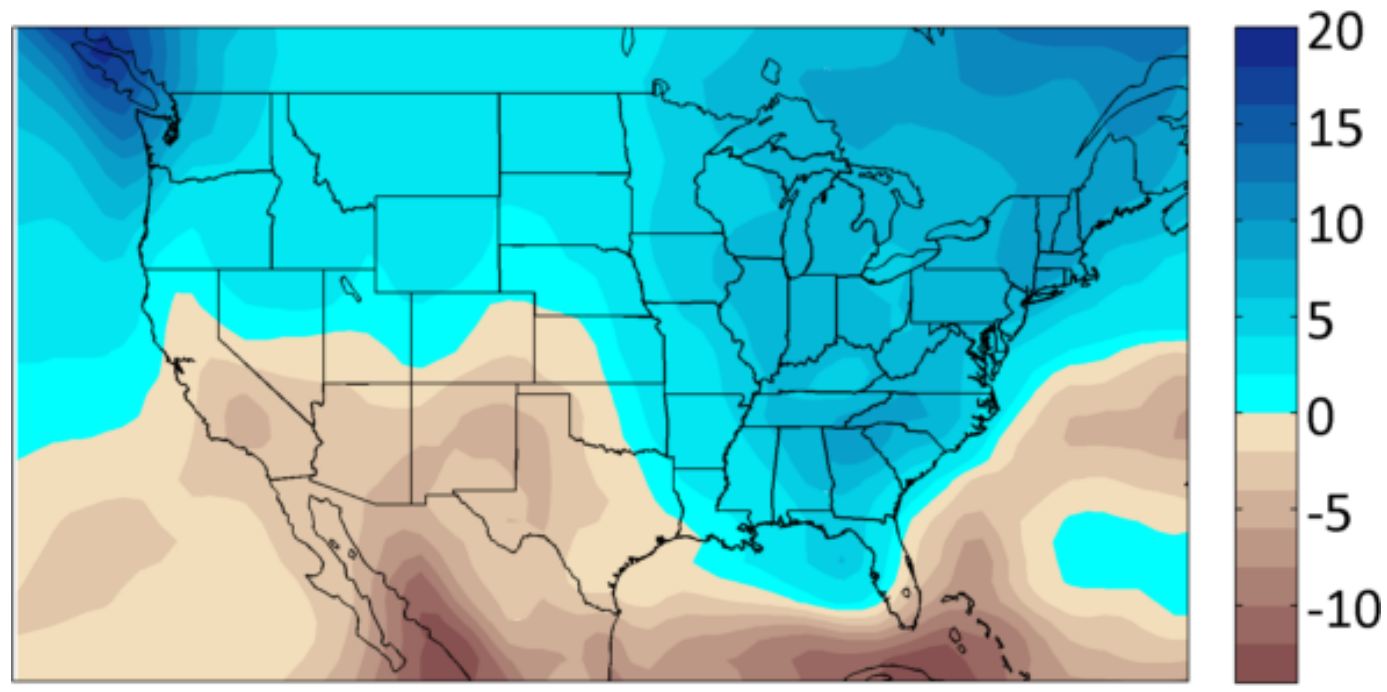

Difference between "Intelligent" and Equal-weight ensemble means (cm/year)

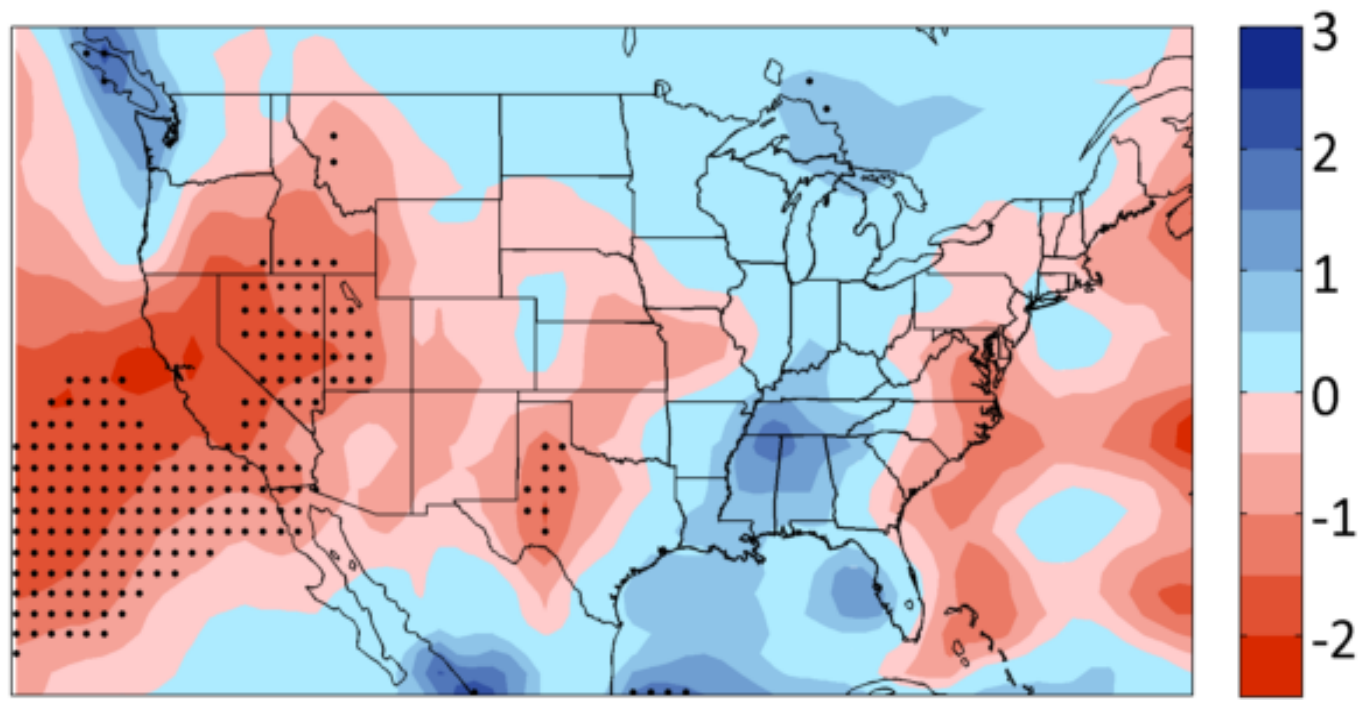




\section{Results: new $21^{\text {st }}$-century projections}

"Intelligent" ensemble mean temperature trend $\left({ }^{\circ} \mathrm{C}\right)$
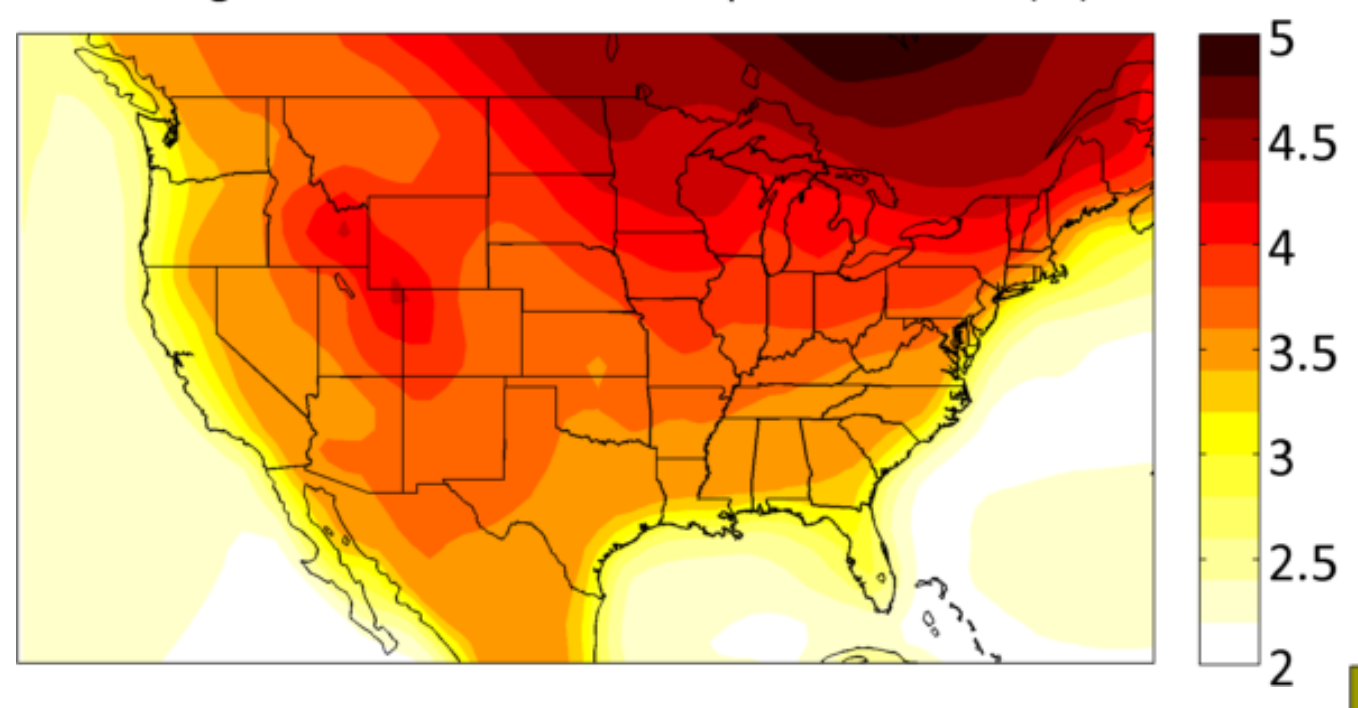

US mean temperature increase: $3.9^{\circ} \mathrm{C}$

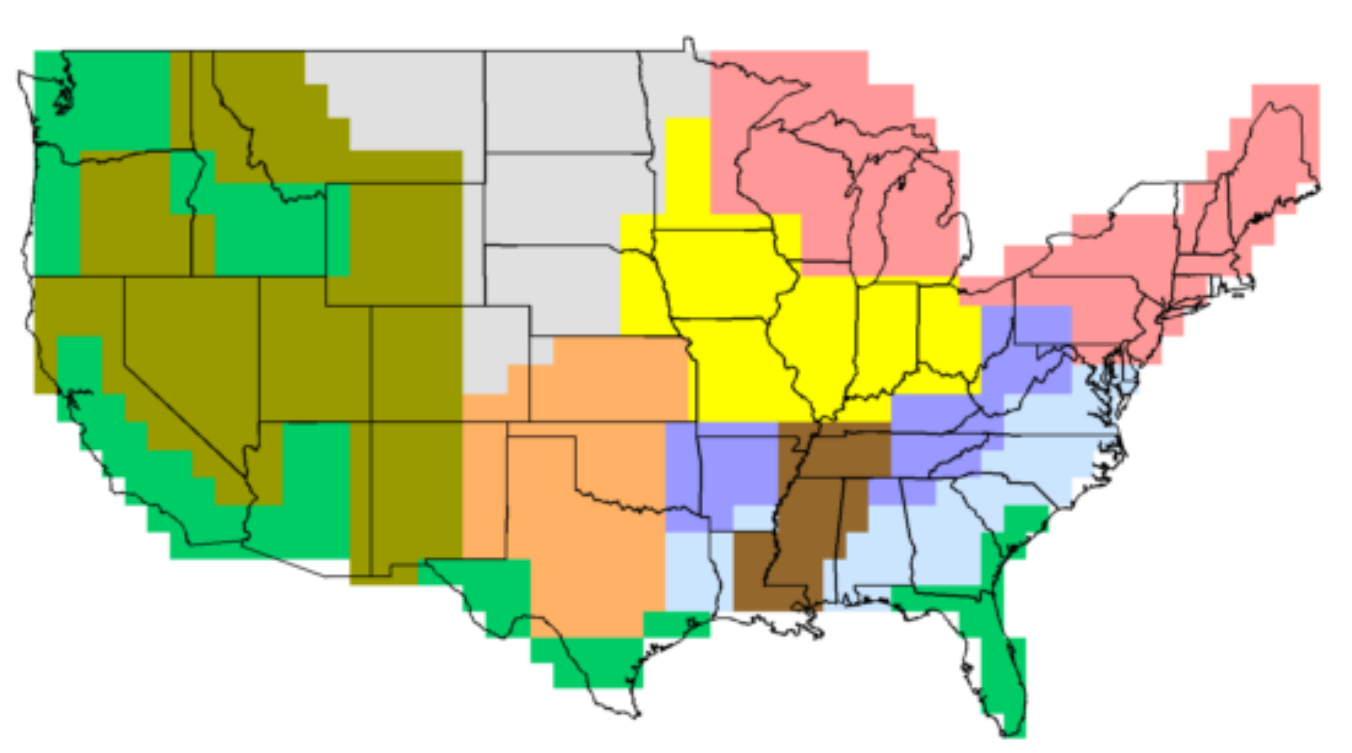

Basin and Range: $3.9^{\circ} \mathrm{C}$ Fruitful Rim: $3.4^{\circ} \mathrm{C}$ Prairie Gateway: $3.8^{\circ} \mathrm{C}$ Northern Great Plains: $4.1{ }^{\circ} \mathrm{C}$ Heartland: $4.1^{\circ} \mathrm{C}$ Northern Crescent: $4.3^{\circ} \mathrm{C}$ Eastern Uplands: $3.8^{\circ} \mathrm{C}$ Southern Seaboard: $3.5^{\circ} \mathrm{C}$ Mississippi Portal: $3.6^{\circ} \mathrm{C}$ 


\section{Results: new $21^{\text {st }}$-century projections}

"Intelligent" ensemble mean precipitation trend (cm/year)
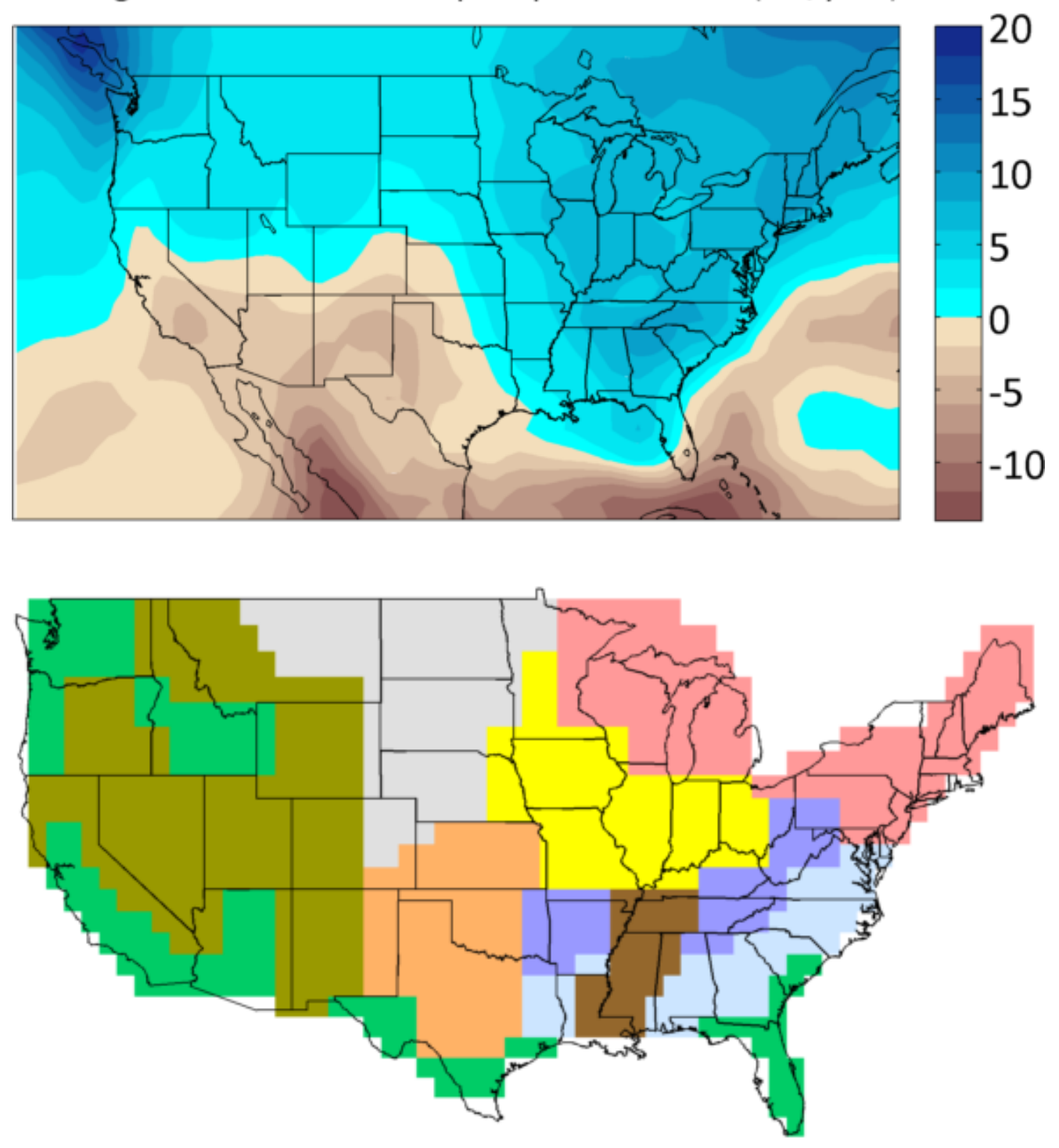

Basin and Range: $0.6 \mathrm{~cm} /$ year

Fruitful Rim: $0.8 \mathrm{~cm} /$ year

Prairie Gateway: $-1.8 \mathrm{~cm} /$ year

Northern Great Plains: $2.7 \mathrm{~cm} /$ year

Heartland: $7.2 \mathrm{~cm} /$ year

Northern Crescent: $9.1 \mathrm{~cm} /$ year

Eastern Uplands: $6.8 \mathrm{~cm} /$ year

Southern Seaboard: $6.8 \mathrm{~cm} /$ year

Mississippi Portal: 5.4 cm/year 16 


\section{Results: new $21^{\text {st }}$-century projections}

"Intelligent" ensemble mean surface shortwave radiation trend $\left(\mathrm{W} / \mathrm{m}^{2}\right)$
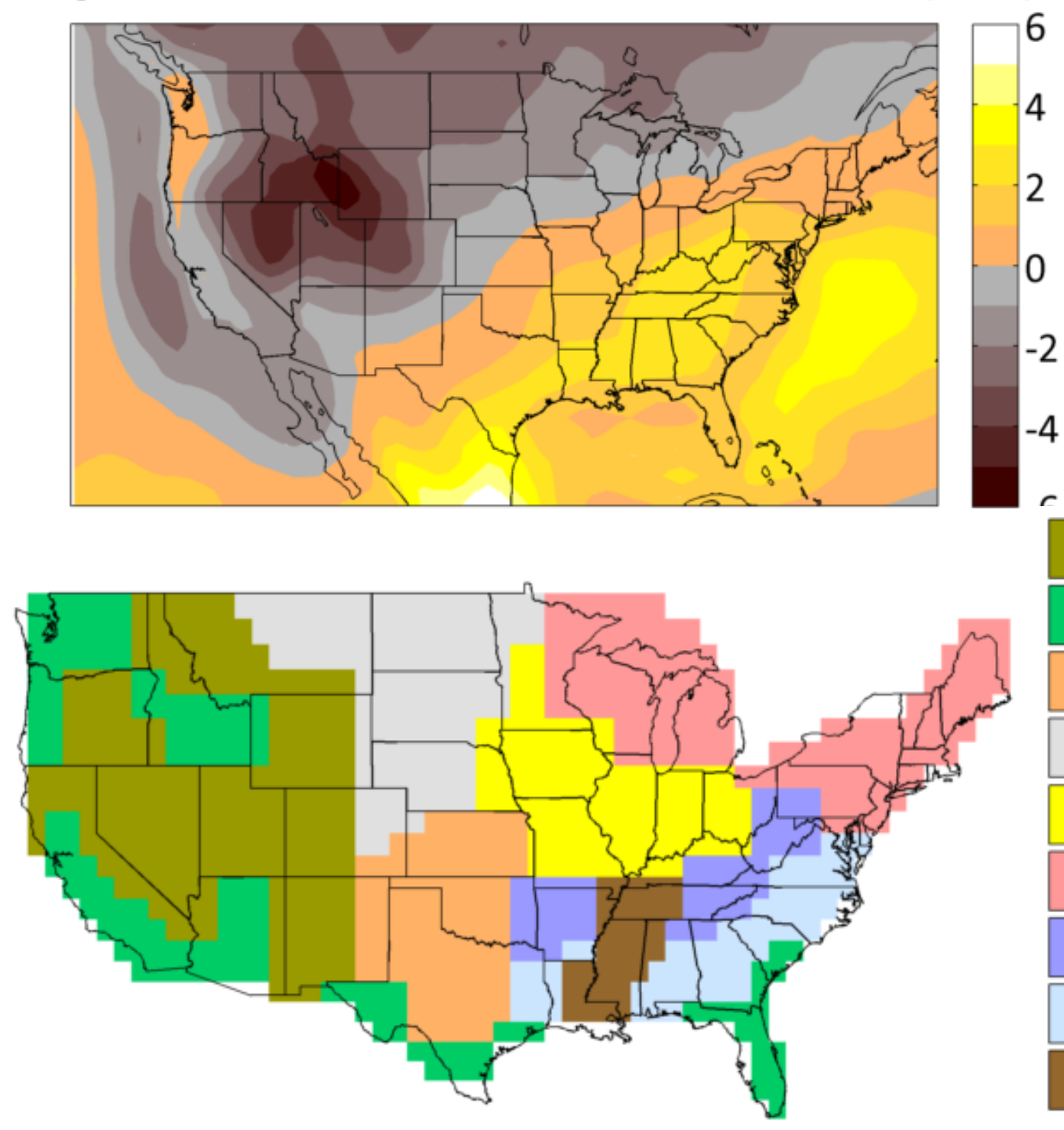

US mean decrease in surface solar radiation: -.33 Watts $/ \mathrm{m}^{2}$

Basin and Range: -2.4 Watts $/ \mathrm{m}^{2}$ Fruitful Rim: -0.5 Watts $/ \mathrm{m}^{2}$ Prairie Gateway: 0.7 Watts $/ \mathrm{m}^{2}$ Northern Great Plains: -1.9 Watts $/ \mathrm{m}^{2}$ Heartland: 0.7 Watts $/ \mathrm{m}^{2}$ Northern Crescent: -0.1 Watts $/ \mathrm{m}^{2}$ Eastern Uplands: 2.7 Watts $/ \mathrm{m}^{2}$ Southern Seaboard: 2.5 Watts $/ \mathrm{m}^{2}$ Mississippi Portal: 2.6 Watts $/ \mathrm{m}^{2}$ 


\section{Summary and Conclusions}

- Data constrained climate change projections are one way science an address society's need for better climate information.

- The "Intelligent" Ensemble method uses model performance to constrain projections.

- The data-constrained projections different from the equal weighted projections by as much as $50 \%$. 